\title{
A novel method for calculating polymer adsorption in fractures through a dual porosity model
}

\author{
Behnam Sedaee $^{1} \cdot$ Assila Taymourtash $^{1}$
}

Received: 27 October 2021 / Accepted: 3 January 2022 / Published online: 18 January 2022

(c) The Author(s) 2022

\begin{abstract}
Increasing demand for energy and limited capacity of available resources of fossil fuels have drawn attention to enhanced oil recovery for maximum use of the available resources. Among different enhanced recovery methods, polymer injection maintains the advantages of water flooding, including the simplicity of this technique, while eliminating some of its drawbacks such as high mobility of the injected water. Therefore, mechanisms involved in the flow of polymer in the reservoirs need to be well understood and modeled. An important phenomenon affecting the flow of polymer in the oil reservoirs is polymer adsorption on reservoir rock. In fractured reservoirs, the polymer is adsorbed both in the matrix and the fracture. Miscalculation of the amount of adsorbed polymer results in the miscalculation of the fracture permeability on one hand, and concentration of dissolved polymer on the other. This, in turn, leads to over- or under-estimation of polymer injection performance in fractured reservoirs. An important tool for simulating fractured reservoirs is dual-porosity models. The models, however, may not correctly estimate polymer adsorption in fractures. This is attributable to the assumptions used in the dual-porosity model and the nature of adsorption. The present study attempts to improve the way this phenomenon is modeled by dual-porosity models. A new parameter called "pseudo-density" is defined here to replace the density of the fracture cells in the dual-porosity model for polymer adsorption. Then, a hypothetical reservoir is simulated using this new parameter, and the results of the two simulations, one with density and the other with pseudo-density, are compared in terms of three parameters, including the polymer adsorbed in the reservoir, the polymer dissolved in the aqueous phase, and the polymer produced in the reservoir. The results show that using pseudo-density instead of rock density decreases the required polymer amount to reach the maximum polymer concentration. Consequently, polymer solution viscosity and permeability reduction are increased. Furthermore, it results in increasing the polymer production compared to the conventional dualporosity models.
\end{abstract}

Keywords Polymer flooding $\cdot$ Fractured reservoirs $\cdot$ Dual-porosity $\cdot$ Adsorption $\cdot$ Pseudo-density

\section{List of symbols}

$S_{\text {frac. }} \quad$ Rock surface in each fracture cell

$S_{\text {mat. }} \quad$ Rock surface in each matrix cell

$V_{\text {cell,frac. }} \quad$ Volume of each fracture cell

$V_{\text {cell,mat. }}$ Volume of each matrix cell

$\mathrm{RM}_{\text {frac. }} \quad$ Rock mass in each fracture cell

$\mathrm{RM}_{\text {mat. }} \quad$ Rock mass in each matrix cell

$V_{\text {void,mat. }}$ Volume of empty space in each matrix cell

$n \quad$ Number of capillary tubes

$K_{\mathrm{f}} \quad$ Fracture permeability

Behnam Sedaee

sedaee@ut.ac.ir

1 Institute of Petroleum Engineering, School of Chemical Engineering, College of Engineering, University of Tehran, Tehran, Islamic Republic of Iran
$M_{\text {frac. }} \quad$ Specific surface area in each fracture cell

$M_{\text {mat. }} \quad$ Specific surface area in each matrix cell

$\bar{r}_{\text {frac. }} \quad$ Average radius of each fracture

$\bar{r}_{\text {mat. }} \quad$ Average radius of capillary tubes

$\mathrm{RV}_{\text {frac. }} \quad$ Rock volume in fracture cell

$\mathrm{RV}_{\text {mat. }} \quad$ Rock mass in matrix cell

$t \quad$ Tortuosity coefficient

$L \quad$ Capillary tube length

$P_{\mathrm{c}} \quad$ Capillary pressure

\section{Greek symbols}

$\rho_{\text {mat. }} \quad$ Rock density in matrix cell

$\rho_{\text {frac. }} \quad$ Rock density in fracture cell

$\sigma \quad$ Surface tension

$\emptyset_{\text {mat. }} \quad$ Matrix porosity

$\emptyset_{\text {frac. }} \quad$ Matrix porosity 


\section{Introduction}

The simplicity of the technique and low cost of water injection have always made it the first choice of improvement to oil recovery methods. In naturally fractured reservoirs (NFRs), due to substantial differences between the matrix and fracture permeability, the injected water tends to flow through the fracture channels and reach the producing well. Therefore, as the level of water in the well increases, it becomes uneconomical to produce oil, resulting in the well-being shut down, even though there is still a significant amount of oil in the matrix. As a result, this method is inefficient when applied to the fractured reservoirs. To increase the efficiency of water injection in fractured reservoirs, the polymer was injected instead of water. Polymer added to injected water can reduce the mobility of the injected solution, causing it to take longer for the injected fluid to reach the producing well.

Based on the available reports, few polymer flooding projects applied to the naturally fractured reservoirs in recent years (Zornes et al. 1986; Manrique et al. 2007; DeHekker 1986; Jamaloei 2011). One of the major reasons for this issue is the difficult estimation of the required polymer for a field project. To estimate the required polymer in a field project, two methods can be implemented, including the laboratory and the numerical simulation methods. The heterogeneous nature of the fractured reservoirs and technical limitations in core recovery from the wells in these fields are the main reasons that limited the laboratory simulations on the polymer flooding in the fractured reservoirs. Due to these limitations, laboratory studies mainly focused on the imbibition process, and other mechanisms of recovery in the naturally fractured reservoirs have been overlooked. (Ghedan and Poettmann 1991; Babadagli 2001a, b, c; Babadagli 2003a, b; Nurkamelia and Arihara 2004). Few implemented core flooding projects were the normal (unfractured) cores that are artificially fractured (Shedid 2006). Therefore, they cannot show the real condition in the field. Due to these limitations in the laboratory studies, numerical simulation of the naturally fractured reservoirs has been developed as well. Regarding the recent improvements in numerical simulation of fractured reservoirs, it is anticipated that the application of this method in real fields could be more feasible and practical when the procedure of polymer injection into these reservoirs is properly simulated.

The first numerical simulation of polymer injection to fractured reservoirs was performed by Gilman et al. in 1983 (Gilman and Kazemi 1983). This model was developed based on the concept of dual-porosity, and was able to simulate various phenomena including polymer injection to the reservoir. The main mechanisms of polymer flow in reservoirs were simulated using appropriate equations, and then the equations were solved numerically. During the next few years, the numerical simulators for fractured reservoirs were completed; however, the polymer flow phenomena described in these reservoirs were still using the equations of Gilman et al.

In 2010, Alajmi et al. studied the procedure of polymer injection to a synthetic fractured reservoir, using simulation through a dual-porosity model (Alajmi 2010). They investigated the effect of injected polymer concentration, the total quantity of the injected fluid, fracture density, and the injection pattern on oil recovery efficiency. They reported that the fracture density has a significant effect on the polymer injection efficiency, in a way that whenever the fracture density is less than a critical quantity, using horizontal well instead of vertical, the efficiency of the polymer injection increases. This study concluded that increasing the polymer concentration and the quantity of injection, improves the polymer injection efficiency compared to the water injection. However, depending on the fracture density this effect could be varied.

Taymourtash et al. (2018), performed polymer injection simulation at two fractured reservoir models including the dual-medium and dual-porosity modeling. The results from both models were compared and the proficiency of the dual-porosity model in the polymer flooding simulation was investigated. The results of this study demonstrated that the assumption of the equal cells volume of matrix and fracture in the dual-porosity model, which has been used in many previous studies, results in inaccurate calculations of the amount of the adsorbed polymer. Consequently, the prediction of the polymer solution behavior in the reservoir is not be reliable using this model. In the case of using the assumption of equal cells volume, some parameters should be modified. Some guidelines for modification of the simulation were also proposed.

As mentioned above, one of the best models to simulate fractured reservoirs is a dual-porosity model. This model has been applied increasingly in recent years, and acceptable correspondence of the results with the behavior of real the reservoirs has clearly demonstrated its effectiveness. However, applying the dual-porosity model in polymer injection simulation has shown a number of errors. In this study, first, the procedure of adsorption simulation in polymer injection in a dual porosity model, and the effective parameters have been investigated. After clarification of the existing errors, a novel technique is introduced and implemented in the modeling of adsorption procedure in the naturally fractured reservoirs through the dual-porosity model. This novel method allows to use the simplifying assumption of the dual-porosity model, without unwanted errors corresponding to the assumption. 
Finally, using a numerical example, the effect of correction of adsorption simulation on the results of a fractured reservoir simulation has been demonstrated. It should be mentioned that this study does not aim to investigate the efficiency of the polymer injection procedure in fractured reservoirs, but investigates the applicability of the dual porosity model in polymer injection simulation.

\section{Problem statement}

Adding polymer to the injected water reduces the mobility through two different mechanisms: increasing the viscosity of the injected fluid, and reducing permeability. The absolute permeability of the reservoir rock decreases due to the reduction in the effective diameter of the rock pores, which occurs as a result of the polymer adsorption on the rock surface. On the other hand, relative permeability reduces due to the attraction between adsorbed polymer molecules and those in the solution. For modeling the polymer flooding, simulators introduce a new parameter, called "adsorbed polymer concentration." This dimensionless parameter identifies the mass of the adsorbed polymer on the unit of the rock mass. There is a direct equilibrium relationship between adsorbed polymer concentration and polymer solution concentration; hence, whenever the concentration of the adsorbed polymer is miscalculated, the calculation of polymer solution concentration will also be incorrect. Therefore, miscalculating adsorbed polymer concentration leads to over or under estimation of the rock permeability and polymer solution viscosity, which results in miscalculation of the polymer injection performance. Also, if the quantity of the adsorbed polymer in fracture is incorrectly calculated, the required quantity of the polymer to be injected to the reservoir will be over or under estimated, which influences the calculation of expenses for the procedure and designing the required equipment. Therefore, the correct calculation of the adsorbed polymer concentration is of outmost importance both from procedural and economical point of view.

In reality, the quantity of the polymer adsorption depends on the rock surfaces, not on the rock mass. But, regarding the fact that the ratio of surface to volume (specific surface area), and mass to volume (density) are fixed in each rock type, the concentration of the adsorbed polymer for each rock-polymer system is measured and calculated per rock mass.

In the commercial numerical simulators, adsorbed polymer concentration is obtained through the adsorption isotherm curve; then the quantity of the adsorbed polymer in each cell is calculated through multiplying the value of the adsorbed polymer concentration (from adsorption isotherm curve) by the rock mass of the corresponding cell. The rock mass in each cell is calculated by multiplying the value of the cell volume by the rock density. It is worth mentioning that adsorption isotherm and rock density are the input data of polymer flooding model.

Regarding this explanation, three parameters are involved to model the quantity of adsorption in fracture, including the rock volume (cell volume), rock density and adsorption curve.

In dual-porosity models, the dimensions of the matrix and fracture cells are assumed to be equal. However, the porosity of the fracture cells is far less than the matrix cells, so that the volume of pores in fracture cells in the model is equal to the volume of pores in the real condition of the reservoir. Since the cell volume is pore volume plus rock volume, rock volume in fracture cells is far more than those of the real condition. For instance, in a typical reservoir where the matrix porosity is $10 \%$, and the fracture porosity is $0.1 \%$, the rock volume in fracture cells is about 1.1 times more than the rock volume in matrix cells, although we know there is no rock mass in the fracture medium in the real reservoir condition.

Since the fluid in the reservoir flows through the pores, this hypothesis causes no problem in flow modeling. However, regarding the fact that modeling the adsorption phenomenon in the dual-porosity model and other models has been carried out based on the rock mass, which is related to rock volume through density, this assumption results in a considerable error in the calculations. Therefore, it is necessary to somehow correct the quantity of the polymer adsorbed in fracture.

To correct the quantity of the adsorbed polymer in fracture cells, one of the three parameters, namely cell volume, rock density or adsorption curve should be corrected. In the dual-porosity model, to avoid divergence in solving the flow equations, the cell volume should be equal in fracture and matrix cells. Thus, there is no possibility of correcting this parameter. Similarly, since the numerical value of adsorbed polymer has a direct influence on calculating the reduction in rock permeability, applying changes on adsorption curve is impossible. Therefore, the only changeable parameter to correct the quantity of adsorption is rock density. The rock mass (the product of rock volume and rock density) can be corrected by changing the value of density, which results in correction of adsorbed polymer quantity (the product of rock mass and adsorbed polymer concentration), without changing the adsorbed polymer concentration.

The essential question to be addressed is that how much the rock density should be reduced, so that the quantity of the adsorbed polymer in a fracture in the model corresponds to the real quantity. In this study, a novel method to correct the density is presented and applied to a synthetic reservoir model to verify the implementation. Performing the simulation using the corrected density, so called pseudo-density,

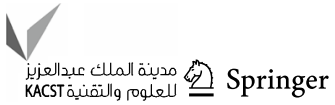


adsorbed polymer in a fracture will be close to the quantity in real condition.

\section{Model improvement}

As explained, pseudo-density, as a rock density, is used to correct the fracture surface adsorption. In order to determine the quantity of the pseudo-density, the ratio of the adsorbed polymer in each fracture cell to adsorbed polymer in each matrix cell, which is calculated through capillary tube model (representative of real reservoir), is equated with the same parameter in the dual-porosity model. Then, the numerical value of pseudo-density is determined from this equality.

The ratio of the adsorbed polymer in each fracture cell to adsorbed polymer in each matrix cell is equal to the ratio of rock surface in each fracture cell to that of each matrix cell, as presented in the following equation:

$\frac{\text { polymer adsorbed in fracture }}{\text { polymer adsorbed in matrix }}=\frac{S_{\text {frac. }}}{S_{\text {mat. }}}=\frac{M_{\text {frac. }} \times V_{\text {cell,frac }}}{M_{\text {mat. }} \times V_{\text {cell,mat }}}$

From the capillary tube model, specific surface area in each matrix cell is as follows:

$M_{\text {mat. }}=\frac{S_{\text {mat. }}}{V_{\text {cell,mat }}}=\frac{n \times 2 \pi \bar{r}_{\text {mat. }} \times L \times t}{V_{\text {cell,mat. }}}$

In the capillary tube model, rock porosity in each cell is calculated through the following equation:

$\emptyset_{\text {mat. }}=\frac{V_{\text {void,mat. }}}{V_{\text {cell,mat. }}}=\frac{n \times \pi \bar{r}_{\text {mat. }}^{2} \times L \times t}{V_{\text {cell,mat }}}$

The combination of Eqs. (2) and (3) leads to:

$M_{\text {mat. }}=\frac{2 \emptyset_{\text {mat. }}}{\bar{r}_{\text {mat. }}}$

In this model, since a bundle of capillary tubes (Amyx et al. 1960) is supposed as rock matrix, the fracture will be taken into account as a tube with the radius of $\bar{r}_{\text {frac. }}$. Similar to the matrix, fracture cells specific surface area is:

$M_{\text {frac. }}=\frac{S_{\text {frac. }}}{V_{\text {cell,frac. }}}=\frac{2 \emptyset_{\text {frac. }}}{\bar{r}_{\text {frac. }}}$

Replacing (4) and (5) in (1) leads to:

$\frac{\text { polymer adsorbed in fracture }}{\text { polymer adsorbed in matrix }}=\frac{S_{\text {frac. }}}{S_{\text {mat. }}}=\frac{\frac{2 \emptyset_{\text {frac }}}{\bar{r}_{\text {frac. }}} \times V_{\text {cell,frac }}}{\frac{2 \emptyset_{\text {mat }}}{\bar{r}_{\text {mat. }}} \times V_{\text {cell, mat }}}$
Since in the dual porosity model, it is assumed that the volume of cells is equal in matrix and fracture, therefore Eq. (6) can be simplified as:

$\frac{\text { polymer adsorbed in fracture }}{\text { polymer adsorbed in matrix }}=\frac{S_{\text {frac. }}}{S_{\text {mat. }}}=\frac{\emptyset_{\text {frac. }} \times \bar{r}_{\text {mat. }}}{\emptyset_{\text {mat. }} \times \bar{r}_{\text {frac. }}}$

As mentioned before, in polymer injection simulation, the ratio of adsorbed polymer in fracture to the adsorbed polymer in the matrix is equal to the ratio of rock mass in each matrix cell to that of each fracture cell. On the other hand, rock mass is equal to the product of rock volume and its density. Consequently, the following equation can be driven:

$\frac{\text { polymer adsorbed in fracture }}{\text { polymer adsorbed in matrix }}=\frac{\mathrm{RM}_{\text {frac. }}}{\mathrm{RM}_{\text {mat. }}}=\frac{\mathrm{RV}_{\text {frac. }}}{\mathrm{RV}_{\text {mat. }}} \times \frac{\rho_{\text {frac. }}}{\rho_{\text {mat. }}}$

Rock volume in each cell is determined by subtracting the volume of cell pores from the cell volume. Therefore, Eq. (7) can be rewritten as follows:

$\frac{\text { polymer adsorbed in fracture }}{\text { polymer adsorbed in matrix }}=\frac{V_{\text {cell,frac }} \times\left(1-\emptyset_{\text {frac. }}\right)}{V_{\text {cell,mat. }} \times\left(1-\emptyset_{\text {mat. }}\right)} \times \frac{\rho_{\text {frac. }}}{\rho_{\text {mat. }}}$

Since the volume of cells in fracture and matrix is equal, Eq. (9) can be simplified as:

$\frac{\text { polymer adsorbed in fracture }}{\text { polymer adsorbed in matrix }}=\frac{\left(1-\emptyset_{\text {frac. }}\right)}{\left(1-\emptyset_{\text {mat. }}\right)} \times \frac{\rho_{\text {frac. }}}{\rho_{\text {mat. }}}$

The Eqs. (7) and (10) result in:

$\frac{\emptyset_{\text {frac. }} \times \bar{r}_{\text {mat. }}}{\emptyset_{\text {mat. }} \times \bar{r}_{\text {frac. }}}=\frac{\left(1-\emptyset_{\text {frac. }}\right)}{\left(1-\emptyset_{\text {mat. }}\right)} \times \frac{\rho_{\text {frac. }}}{\rho_{\text {mat. }}}$

By rewriting Eq. (11) for fracture density, a new value for this parameter is obtained. Since from a physical point of view, the rock density is equal in fracture and matrix, the density, which is obtained here is called "pseudo-density," and replaces the real density in modeling procedure. In order to determine the pseudo-density, (11) is rewritten as follows:

$\rho_{\text {frac. }}=\frac{\left(1-\emptyset_{\text {frac. }}\right)}{\left(1-\emptyset_{\text {mat. }}\right)} \times \frac{\emptyset_{\text {frac. }}}{\emptyset_{\text {mat. }}} \times \frac{\bar{r}_{\text {mat. }}}{\bar{r}_{\text {frac. }}} \times \rho_{\text {mat. }}$

where the value of porosity in the fracture and matrix are input data of the model, but the average radius of pores in fracture and matrix should be calculated through appropriate equations.

The average radius of matrix cells is calculated through capillary pressure as (Amyx et al. 1960): 
$\bar{r}_{\text {mat. }}=\frac{2 \sigma}{P_{\mathrm{c}}}$

The capillary pressure and surface tension are obtained by performing the experiment in each fluid system. The average radius of fracture cells is determined through fracture permeability as follows (Van Golf Rotch 1982):

$\bar{r}_{\text {frac }}=\sqrt{12 K_{\mathrm{f}}}$

where $K_{\mathrm{f}}$ is used as entry data in the simulation.

By placing the average radius of cells in the matrix and fracture, pseudo-density is calculated through the above equations. Therefore, in a simulation study, whenever rock density in fracture cells is replaced with pseudo-density, the quantity of adsorbed polymer will be closer to the quantity in the real condition.

\section{Numerical example}

In order to evaluate the proposed modified method, a numerical simulation has been carried out on a hypothetical reservoir. The results of this simulation demonstrate both the importance of this issue and the validity of the proposed method.

The synthetic reservoir is a horizontal fractured reservoir with dimensions of $800 \times 3200 \times 3200 \mathrm{ft}^{3}$. This reservoir contains 27 matrix blocks, where the fractures have been placed between each two matrix blocks, based on the dualporosity model assumption. The overall scheme of the aforementioned hypothetical reservoir is shown in Fig. 1.

In this constructed reservoir model, the values of porosity in the matrix and fracture are 10 and $0.1 \%$, respectively, and matrix and fracture permeabilities are 10 and $1000 \mathrm{md}$, respectively. The fluid in the reservoir is an undersaturated oil with the viscosity of $10 \mathrm{cp}$, in the temperature and pressure of the reservoir. The reservoir temperature is $600 \mathrm{R}$, and its primary pressure is $3200 \mathrm{psi}$. The primary saturation of the water in the reservoir is $30 \%$, which is equal to the saturation amount of irreducible water. Therefore, before fluid injection, there is no

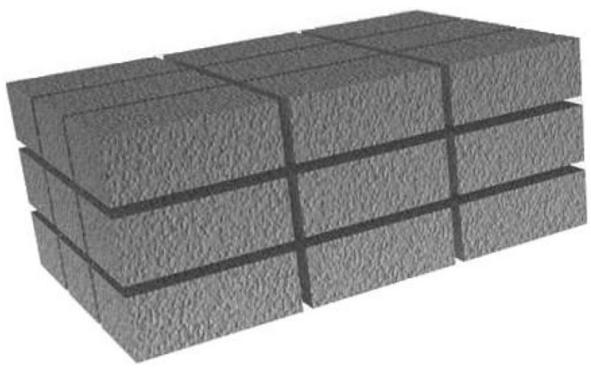

Fig. 1 Schematic of the simulated reservoir mobile water phase in the reservoir. The constructed reservoir model contains an injection well and a production well. In this reservoir, the production and injection have been simulated for 25 years with the same rate of 1000 STB/day.

The primary concentration of the injected polymer solution to the reservoir is $1000 \mathrm{ppm}$ with the viscosity of $10 \mathrm{cp}$.

Regarding the fact that when the polymer solution flows through the reservoir, some of the dissolved polymer is adsorbed by the rock and so, the concentration of polymer solution changes in the reservoir. Figures 2 and 3 represent the models defined for the polymer solution viscosity and polymer adsorption, both as a function of polymer solution concentration.

The most important specifications of the hypothetical reservoir used in the calculation of pseudo-density are as follows:

$P_{\mathrm{c}}=12 \mathrm{psi}=8.2740 \times 10^{4} \mathrm{~N}$

$\sigma=25 \frac{\text { dynes }}{\mathrm{cm}}=25 \times 10^{-3} \frac{\mathrm{N}}{\mathrm{m}}$

$K_{\mathrm{f}}=1000 \mathrm{~m} D=0.987 \times 10^{-12} \mathrm{~m}^{2}$

$\rho_{\text {mat. }}=950 \mathrm{lb} / \mathrm{bbl}$

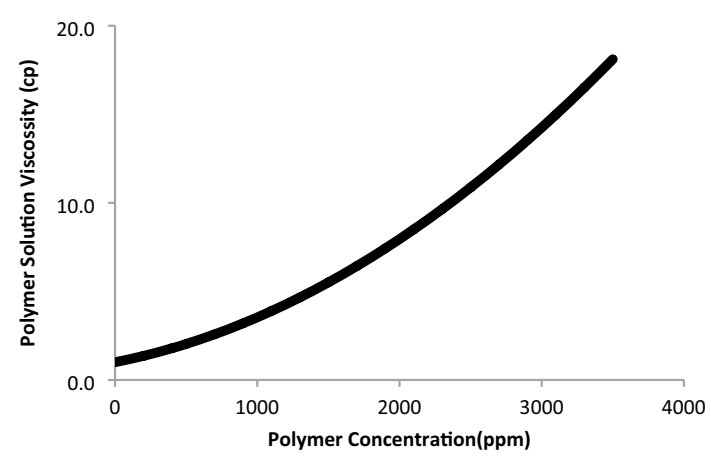

Fig. 2 Polymer solution viscosity vs. polymer concentration

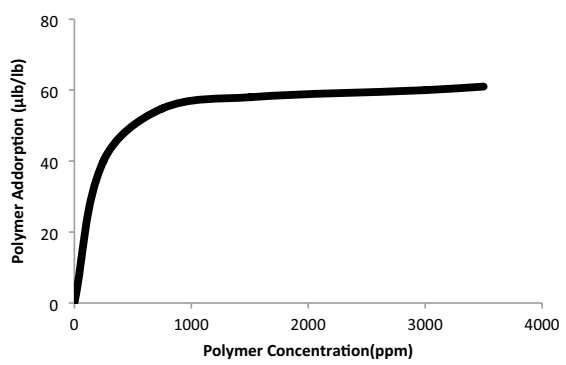

Fig. 3 Adsorption isotherm vs. polymer concentration

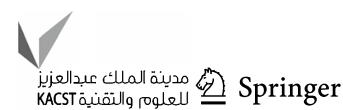


In order to determine the pseudo-density, first, the average radius of matrix cavities, and fracture radius should be calculated according to the Eqs. (12) and (13):

$\bar{r}_{\text {mat. }}=\frac{2 \sigma}{P_{\mathrm{c}}}=\frac{2 \times 25 \times 10^{-3}}{8.2740 \times 10^{4}}=6.04 \times 10^{-7} \mathrm{~m}$

$\bar{r}_{\text {frac }}=\sqrt{12 K_{\mathrm{f}}}=\sqrt{12 \times 0.987 \times 10^{-12}}=3.44 \times 10^{-6} \mathrm{~m}$

Finally, pseudo-density is determined using Eq. (11):

$\rho_{\text {frac. }}=\frac{\left(1-\varphi_{\text {mat. }}\right)}{\left(1-\varphi_{\text {frac. }}\right)} \times \frac{\varphi_{\text {frac. }}}{\varphi_{\text {mat. }}} \times \frac{\bar{r}_{\text {mat. }}}{\bar{r}_{\text {frac. }}} \times \rho_{\text {mat. }}$

$=\frac{(1-0.1)}{(1-0.001)} \times \frac{0.001}{0.1} \times \frac{6.04 * 10^{-7}}{3.44 * 10^{-6}} \times 950 \frac{\mathrm{lb}}{\mathrm{bbl}}=1.51 \mathrm{lb} / \mathrm{bbl}$

From this point, the value of pseudo-density which has been obtained here, substitutes the value of the density in the simulated model of the synthetic reservoir. Then, the results of the two simulations, one with density and the other with pseudo-density, were compared.

\section{Results and discussion}

There are four parameters which affect polymer balance in a reservoir: injected, dissolved, adsorbed and produced polymer. The injected polymer is the entry of the simulation which is a known quantity. On the other hand, there are two equations in order to explain polymer behavior in the reservoir: one is the mass balance and the other is the equilibrium equation between dissolved and adsorbed polymer. Therefore, there is only one unknown in this study and the behavior of other variables will be explained regarding the available equations.

Since changes of the rock density in fracture cells directly affect the quantity of adsorbed polymer, adsorbed polymer has been considered as the independent variable. Then, the behavior of dissolved and produced polymer has been analyzed taking the two available equations into account.

\section{Adsorbed polymer}

Two parameters should be analyzed when studying the adsorbed polymer: one is the quantity and the other is the concentration of the adsorbed polymer. Analyzing the curve of adsorbed polymer quantity in fractures (Fig. 4) shows that in the density model, the quantity of adsorbed polymer in fractures is 300 times more than that of the pseudo-density model, and this quantity is constantly increasing in density model, whereas in the pseudo-density model, the quantity of adsorbed polymer has reached its maximum and remains fixed thereafter.

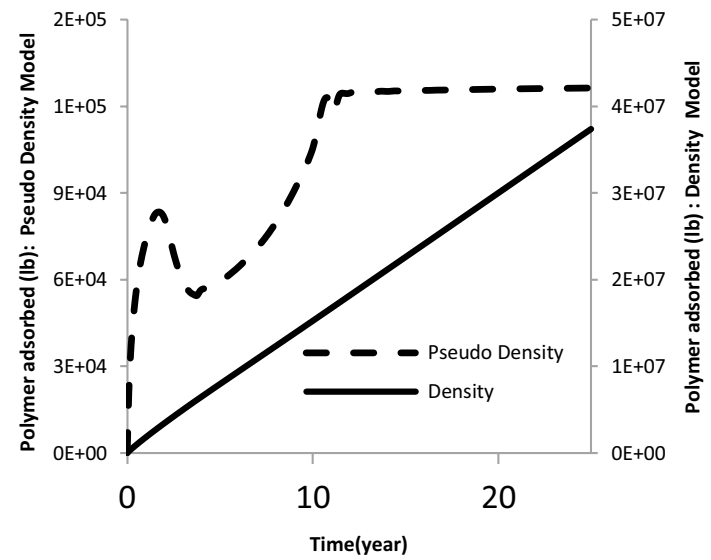

Fig. 4 Comparison of adsorbed polymer quantity in fractures

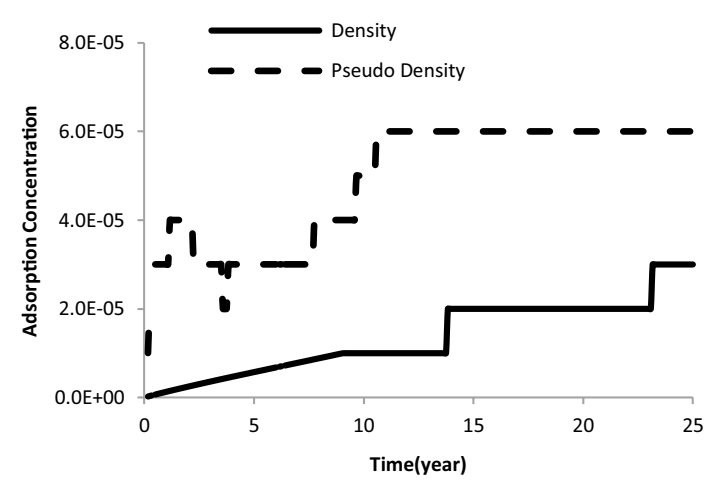

Fig. 5 Comparasion of adsorption concentration

It should be noted that although the curve of adsorbed polymer in pseudo-density model shows a lower quantity, the concentration of the adsorbed polymer is higher, as represented in Fig. 5. The reason is that the concentration of the adsorbed polymer depends on the rock mass in fracture, which in turn relates to the rock volume, which is equal in both models, through density. Replacing density by pseudo-density leads to rock mass reduction, and consequently increases the concentration of the adsorbed polymer (Fig. 5). On the other hand, we know that reduction in the rock permeability in reservoir depends on the adsorbed polymer concentration rather than its quantity. Therefore, rock permeability is more reduced in the pseudo-density model compared to the density model (Fig. 6).

\section{Dissolved polymer}

According to the adsorption isotherm, polymer solution concentration corresponds to the concentration of the adsorbed polymer. Thus, replacing density with pseudo-density results in increasing the concentration of the polymer solution in 


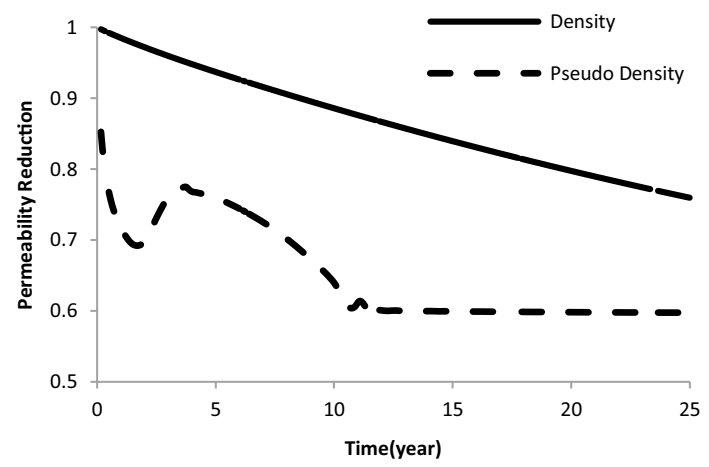

Fig. 6 Comparasion of permeability reduction

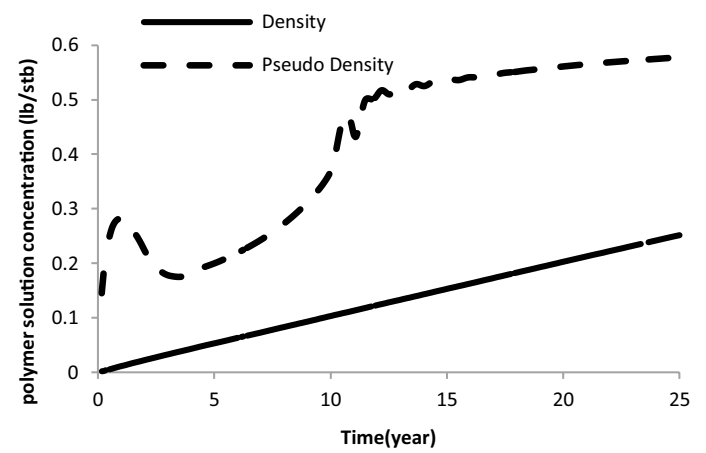

Fig. 7 Comparison of average concentration of polymer solution in fracture cells

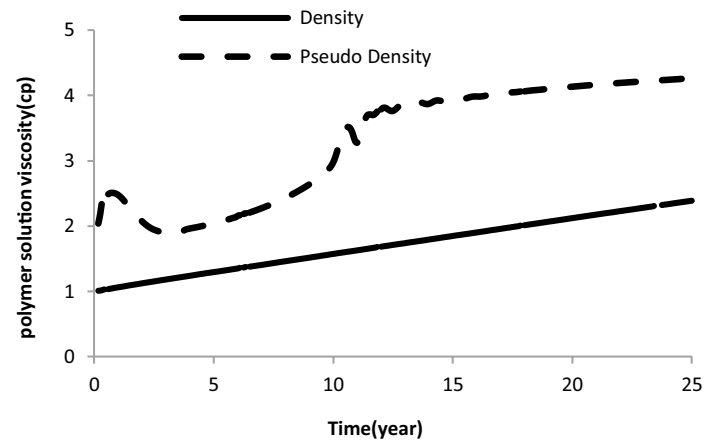

Fig. 8 Comparison of average viscosity of polymer solution in fracture cells

fracture cells in the reservoir. Figure 7 shows the average concentration of the polymer solution in fracture cells during 25 years. An increase in polymer solution concentration leads to the increase in polymer solution viscosity. Therefore, replacing density with pseudo-density in dual porosity model, results in the increase in solution viscosity at fracture cells. Figure 8 shows the average viscosity of the polymer solution in fracture cells in the reservoir. As it can be seen, the quantity of available polymer in solution phase increases

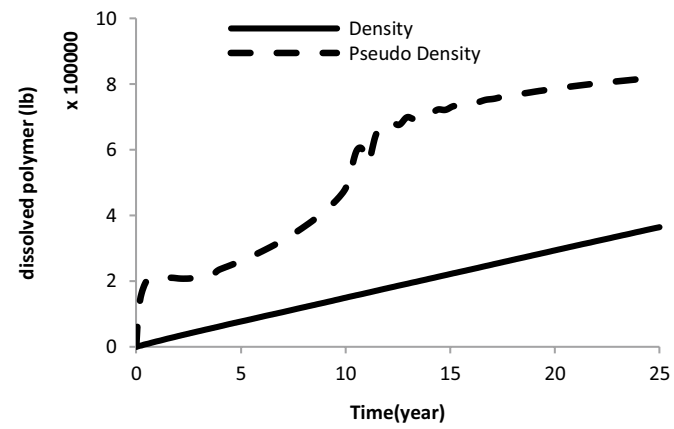

Fig. 9 Comparison of quantity of dissolved polymer in fracture cells

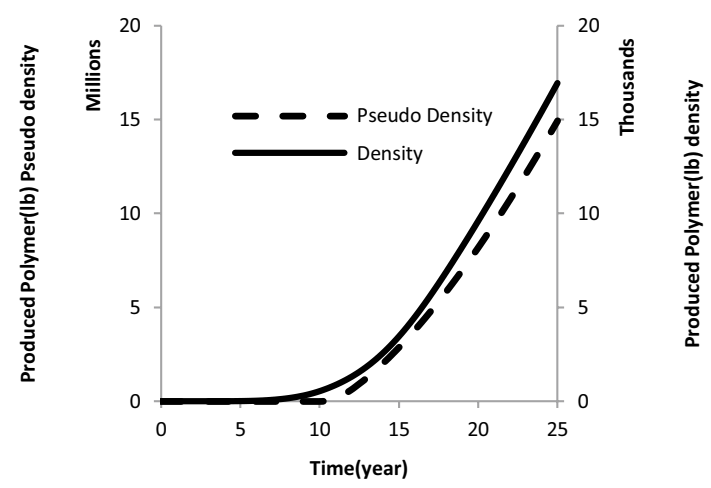

Fig. 10 Comparison of the quantity of producing polymer

in pseudo-density model. Since the volume of pores in both models is equal, if the concentration of dissolved polymer in pseudo-density model increases, the quantity of dissolved polymer in this model will increase accordingly, as shown in Fig. 9.

\section{Produced polymer}

According to the mass balance equation, the quantity of producing polymer equals injected polymer minus the sum of adsorbed and dissolved polymer. As explained, replacing density with pseudo-density increases the quantity of both the adsorbed and dissolved polymer. Therefore, the quantity of the produced polymer is reduced (Fig. 10). The quantity of produced polymer is significantly important from the aspect of polymer injection design, recycling produced polymer, and the economical evaluation of the process.

\section{Conclusion}

Adsorption modeling based on the rock mass, as a common approach in the dual-porosity model, does not correctly reflect the process of polymer adsorption in fracture cells. 
A new parameter called "pseudo-density" was introduced here to replace density with the aim of improving polymer adsorption modeling in fracture cells. Comparing the simulation results for two models, one with density and the other with pseudo-density, in terms of the quantities of the polymer adsorbed in the reservoir, the dissolved polymer, and the produced polymer indicated that:

(1) By modifying polymer adsorption in the fracture using pseudo-density, a smaller quantity of polymer is required to reach maximum concentration of the adsorbed polymer, and this provides rapid minimization of the permeability.

(2) Due to reduced rock volume, as a result of replacing density with pseudo-density, the adsorbed and dissolved polymer form a state of equilibrium at a higher concentration compared to the model which uses density for simulation. Therefore, the viscosity of the solution is also greater than the corresponding value in the density-based simulation.

(3) As polymer adsorption in the fracture cell decreases in the pseudo-density model, greater quantities of polymer can reach the production well through the fracture network. Here, permeability is smaller compared to the unmodified model and the viscosity of the polymer solution is greater than the viscosity in the original model.

(4) In general, the error resulting from the density-based modeling of polymer adsorption in fracture leads to the miscalculation of permeability, viscosity of the polymer solution in fracture, and quantity of the polymer produced in the reservoir. This error can be avoided by replacing density with the new pseudo-density parameter introduced in this study.

Funding No funding was secured for this manuscript.

\section{Declarations}

Conflict of interest On behalf of all the co-authors, the corresponding author states that there is no conflict of interest.

Open Access This article is licensed under a Creative Commons Attribution 4.0 International License, which permits use, sharing, adaptation, distribution and reproduction in any medium or format, as long as you give appropriate credit to the original author(s) and the source, provide a link to the Creative Commons licence, and indicate if changes were made. The images or other third party material in this article are included in the article's Creative Commons licence, unless indicated otherwise in a credit line to the material. If material is not included in the article's Creative Commons licence and your intended use is not permitted by statutory regulation or exceeds the permitted use, you will need to obtain permission directly from the copyright holder. To view a copy of this licence, visit http://creativecommons.org/licenses/by/4.0/.

\section{References}

Alajmi F, Gharbi R, Chase R (2010) The performance of polymer floods in partially fractured reservoirs. J Porous Media 13(11):961-971

Amyx J, Bass D, Whiting RL (1960) Petroleum reservoir engineering physical properties. McGraw-Hill Companies

Babadagli T (2003a) Evaluation of EOR methods for heavy-oil recovery in naturally fractured reservoirs. J Petrol Sci Eng 37:25-37

Babadagli T (2003b) Selection of proper enhanced oil recovery fluid for efficient matrix recovery in fractured oil reservoirs. Colloids Surf A Physicochem Eng Aspects 223:157-175

Babadagli T (2001c) Scaling of cocurrent and countercurrent capillary imbibition for surfactant and polymer injection in naturally fractured reservoirs. SPE J 6(4):465-478

Babadagli T (2001a) Recovery of heavy matrix oil by capillary imbibition in naturally fractured reservoirs. In: SPE international thermal operations and heavy oil symposium, Porlamar, Margarita Island, Venezuela, 12-14 Mar 2001

Babadagli T (2001b) Selection of proper EOR method for efficient matrix recovery in naturally fractured reservoirs. IN: SPE Latin American and Caribbean petroleum engineering conference. Buenos Aires, Argentina, 25-28 Mar 2001

DeHekker TG, Bowzer JL, Coleman RV, Bartos WB (1986) A progress report on polymer-augmented waterflooding in wyoming's North Oregon Basin and Byron Fields. In: SPE enhanced oil recovery symposium. Tulsa, Oklahoma, USA, 20-23 Apr 1986

Ghedan G, Poettmann H (1991) Effect of polymers on the imbibition process: a laboratory study. SPE Reserv Eng 6(1):84-90

Gilman JR, Kazemi H (1983) Improvements in simulation of naturally fractured reservoirs. Soc Pet Eng J 23(4):695-707

Jamaloei BY (2011) Chemical flooding in naturally fractured reservoirs: fundamental aspects and field-scale practices. Oil Gas Sci Technol 66(6):991-1004

Manrique EJ, Muci VE, Gurfinkel ME (2007) eor field experiences in carbonate reservoirs in the United States. SPE Reservoir Eval Eng 10(6):667-686

Nurkamelia, Arihara N (2004) Analysis of spontaneous capillary imbibition for improved oil recovery. In: SPE Asia pacific oil and gas conference and exhibition. Perth, Australia, 18-20 Oct 2004

Shedid SA (2006) Influences of fracture orientation on oil recovery by water and polymer flooding processes: an experimental approach. J Petrol Sci Eng 50:285-292

Taymourtash A, Sedaee B (2018) investigation of applicability of dualporosity model for polymer flooding simulation. J Porous Media 21(13):1379-1393

Van Golf-Racht TD (1982) Fundamentals of fractured reservoir engineering. Elsevier Scientific Publishing Company

Zornes DR, Cornelius AJ, Long HQ (1986) An overview and evaluation of the North Burbank unit block a polymer flood project, Osage County, Oklahoma. International meeting on petroleum engineering. Beijing, China, 17-20 Mar 1986

Publisher's Note Springer Nature remains neutral with regard to jurisdictional claims in published maps and institutional affiliations. 